\title{
COMPARISON AND EXISTENCE RESULTS FOR EVOLUTIVE NON-COERCIVE FIRST-ORDER HAMILTON-JACOBI EQUATIONS
}

\author{
Alessandra Cutrì ${ }^{1}$ And Francesca DA LiO ${ }^{2}$
}

\begin{abstract}
In this paper we prove a comparison result between semicontinuous viscosity subsolutions and supersolutions to Hamilton-Jacobi equations of the form $u_{t}+H(x, D u)=0$ in $\mathbb{R}^{n} \times(0, T)$ where the Hamiltonian $H$ may be noncoercive in the gradient $D u$. As a consequence of the comparison result and the Perron's method we get the existence of a continuous solution of this equation.
\end{abstract}

Mathematics Subject Classification. 70H20, 49L25, 35B05.

Received September 6, 2005. Revised March 24, 2006.

Published online June 5, 2007.

\section{INTRODUCTION}

In this paper we are interested in first order equations

$$
\begin{cases}u_{t}+H(x, D u)=0 & \text { in } \mathbb{R}^{N} \times(0, T) \\ u(x, 0)=h(x) & \text { in } \mathbb{R}^{N},\end{cases}
$$

where the solution $u$ is a real-valued function defined in $\mathbb{R}^{N} \times[0, T), D u$ denotes its gradient and $h$ is a given initial condition, the Hamiltonian $H: \mathbb{R}^{2 N} \rightarrow \mathbb{R}$ is a continuous function. We will prove comparison and existence results under the following structure conditions on the Hamiltonian $H$ :

(H1)

$$
H(x, p)=\Phi\left(H_{0}(x, p)\right)
$$

where $H_{0}: \mathbb{R}^{2 N} \rightarrow \mathbb{R}$ is a continuous function such that for all $R>0$ there is a constant $L_{R}>0$ such that

$$
\left|H_{0}(x, p)-H_{0}(y, p)\right| \leq L_{R}|x-y|
$$

for all $|x|,|y| \leq R,|p| \leq 1$

$$
p \rightarrow H_{0}(x, p) \text { is convex, } H_{0}(x, p) \geq 0, \quad H_{0}(x, \lambda p)=|\lambda| H_{0}(x, p),
$$

Keywords and phrases. Hamilton-Jacobi equations, sub-Riemannian metric, viscosity solution, comparison principle.

${ }^{1}$ Dipartimento di Matematica, Università "Tor Vergata", via della Ricerca Scientifica, 00133 Roma, Italy; cutri@mat .uniroma2 . it

2 Dipartimento di Matematica Pura e Applicata, Università di Padova, via Belzoni 7, 35131 Padova, Italy.

(c) EDP Sciences, SMAI 2007 
for all $x, p \in \mathbb{R}^{N}$ and for all $\lambda \in \mathbb{R}$. Concerning the function $\Phi:[0,+\infty) \rightarrow[0,+\infty)$ we assume that

$$
\Phi \text { is convex, non decreasing, } \Phi(0)=0 \text {. }
$$

(H2) There is $C>0$ such that

$$
H_{0}(x, p) \leq C H_{1}(x, p),
$$

where $H_{1}: \mathbb{R}^{2 N} \rightarrow \mathbb{R}$ is a continuous function satisfying (0.3), (0.4) and such that, for every $y \in \mathbb{R}^{N}$, the stationary eikonal-type equation

$$
H_{1}(x, D d)=1 \quad x \in \mathbb{R}^{N} \backslash\{y\} \quad d(y)=0
$$

has a continuous viscosity solution $d_{y}(x)=d(x ; y) \geq 0$ verifying

$$
\begin{gathered}
\lim _{|x-y| \rightarrow+\infty} d(x ; y)=+\infty, \\
d(x ; y)+d(y ; z) \geq d(x ; z) \quad \text { for all } x, y, z \in \mathbb{R}^{N},
\end{gathered}
$$

and

$$
d(x ; y) \leq K|x-y|^{\alpha} \quad \text { for all } x, y \in \mathbb{R}^{N},|x-y| \leq R,
$$

for some $K, R>0, \alpha \in(0,1]$.

Before proceeding, let us mention that Capuzzo Dolcetta and Ishii (see [14-16]) provide a Hopf-type representation formula for a solution of (0.1) assuming $(\mathbf{H 1})$ and that $(0.6)$ is verified by $H_{0}$ (that is for the particular choice $H_{1}=H_{0}$ and $C=1$ ). The comparison principle we prove here, states that the Hopf-type formula is in fact the unique solution of $(0.1)$, in the case where $H_{1}=H_{0}$.

We would like to premise some comments on the hypothesis (H2), in particular on the condition (0.6). To this purpose we consider the following model case

$$
H(x, p)=\frac{1}{\beta}\left|b^{T}(x) p\right|^{\beta},
$$

where $\beta>1$ and $b$ is a real $N \times M$ matrix-valued locally Lipschitz continuous function. We note that the case $\beta=2$ arises in the study of deterministic unbounded control problems. The hypothesis (H1) obviously holds true in this case; we list below some sufficient conditions on $b(x)$ guaranteeing the validity of the condition $(0.6)$.

(i) The matrix $b$ is bounded: this case has already been treated in several papers (see e.g. [18] and the references therein) and corresponds to the choice $H_{0}(x, p)=\left|b^{T}(x) p\right|, H_{1}(x, p)=|p|$. In this situation the solution of $(0.6)$ is $d(x ; y)=|x-y|$.

(ii) The matrix $b(x) b^{T}(x)$ is uniformly elliptic: in this situation, we can choose $H_{1}=H_{0}$ and the solution $d(x)$ is the Riemannian distance associated to $b(x) b^{T}(x)$, see $e . g$. [3].

(iii) The matrix $b$ is degenerate and possibly unbounded: the main example is when $b^{T}(x)$ is a $C^{\infty}$ function satisfying the Hörmander rank condition, namely for all $x \in \mathbb{R}^{N}$ the Lie algebra generated by $X_{i}=\sum_{j=1}^{N} b_{i j}^{T}(x) \frac{\partial}{\partial x_{j}},(i=1, \ldots, M)$ satisfies:

$$
\operatorname{rank} \operatorname{Lie}\left(X_{1}, \ldots, X_{M}\right)(x)=N
$$

and the following one

$$
\text { (A1) } \begin{aligned}
& \text { For some constant } L>0:(b(x) w-b(y) w) \cdot(x-y) \leq L|x-y|^{2} \\
& \forall x, y \in \mathbb{R}^{N}, \forall w \in \bar{B}(0,1) .
\end{aligned}
$$

In this situation, one can choose $H_{1}=H_{0}$ and $d(x ; y)$ is the intrinsic distance defined in the next section, which is finite in the whole space, satisfies $(0.7),(0.8)$ and (0.9) with $\alpha=\frac{1}{m}, m$ being the maximal length of the 
commutators needed to span the tangent bundle $T\left(\mathbb{R}^{N}\right)$ at each point $x \in \mathbb{R}^{N}$. We remark that the condition $b^{T} \in C^{\infty}$ can be relaxed owing to the "generalized Chow theory" for non smooth vector fields, developed by Rampazzo and Sussmann in [29]. With this regard we propose the following

Example 0.1. We consider the Lipschitz continuous matrix

$$
b^{T}(x)=\left(\begin{array}{lll}
1 & 0 & f\left(x_{1}\right) \\
0 & 1 & f\left(x_{1}\right)
\end{array}\right)
$$

with $f\left(x_{1}\right)=-2 x_{1} \chi_{(-\infty, 0)}-x_{1} \chi_{(0,+\infty)}$, where $\chi_{E}$ stands for the characteristic function of the set $E$. In this case, the "generalized Lie bracket" between the vectors constructed with the rows of $b^{T}$ at the point $x \in \mathbb{R}^{3}$ is the vector

$$
(0,0,-2) \text { if } x_{1}<0, \quad(0,0,-1) \text { if } x_{1}>0,
$$

the multivalued vector function $(0,0,[-2,-1])$ if $x_{1}=0$.

Hence the two rows of $b^{T}$, together with the Lie bracket they generate, span the tangent bundle $T\left(\mathbb{R}^{3}\right)$ at each point $x \in \mathbb{R}^{3}$. Indeed, if $x_{1} \neq 0$, this obviously holds true. At the points $\left(0, x_{2}, x_{3}\right) \in \mathbb{R}^{3}$, the Lie bracket is given by $(0,0, p)$, with $p \in[-2,-1]$. Thus $b^{T}$ is a matrix which satisfies the generalized Hörmander-Chow rank condition of order two and this implies the existence of the solution of (0.6) satisfying (0.7)-(0.9).

Finally we remark that in the model case (0.10) a sufficient condition for (H2) to hold is the existence of a locally Lipschitz continuous matrix $\sigma(x)$ verifying (A1), such that $\sigma(x) \sigma^{T}(x)$ satisfies the Hörmander rank condition, and moreover, for some constant $C>0$

$$
C \sigma(x) \sigma^{T}(x)-b(x) b^{T}(x) \geq 0 \text { for all } x \in \mathbb{R}^{N} .
$$

In this case, one can choose $H_{1}(x, p)=\left|\sigma^{T}(x) p\right|$. It is worth observing that condition (0.11) is always satisfied with $C=1$, by choosing

$$
\sigma(x) \sigma^{T}(x)=\operatorname{diag}\left(\lambda_{1}(x)+1, \ldots, \lambda_{N}(x)+1\right),
$$

where $\lambda_{i}(x)$ for $i=1, \ldots, N$ denote the non negative eigenvalues of the symmetric matrix $b(x) b^{T}(x)$.

The main goal of this paper is to prove a comparison result between unbounded semicontinuous viscosity subsolutions and supersolutions to the problem (0.1). Such a result is beyond the classical results for viscosity solutions (see e.g. $[3,5]$ ) because of the unboundedness both of the solutions and the Hamiltonians. In fact, most of the comparison and uniqueness results in the literature require that either the solutions are uniformly continuous or the Hamiltonian is uniformly continuous with respect to the gradient uniformly in $x$, (see e.g. [6,7]). We recall that uniqueness and existence results of unbounded solutions to a class of first order Hamilton Jacobi equations (corresponding to unbounded control problems which include the case of quadratic Hamiltonians), have been addressed by several authors, see, e.g. the book of Bensoussan [9], the papers of Alvarez [1], Bardi and Da Lio [4], Cannarsa and Da Prato [13], Rampazzo and Sartori [28] in the case of convex operators, and the papers of Da Lio and McEneaney [19] and Ishii [23] for more general operators. However most of these last results have been obtained under assumptions implying the coercivity of the Hamiltonian with respect to the gradient uniformly in the state variable, namely $H(x, p) \rightarrow+\infty$ as $|p| \rightarrow+\infty$ for all $x \in \mathbb{R}^{N}$. With this regard we refer the reader to Barles [5] for the role of the coercivity condition in the unbounded case. Let us only recall that it allows in general to prove the Lipschitz continuity of the subsolutions of (0.1). In the particular case of equation (0.10) the typical assumptions on the matrix $b$ is that either it is bounded or $b(x) b^{T}(x)$ is not singular.

The main contribution of this note is that we get a comparison result by removing the coercivity condition. Somehow this condition is replaced by assuming that (0.6) has a solution $d$ satisfying (0.7)-(0.9).

The method we use in proving the Comparison Theorem is similar in the spirit to the one applied by Ishii in [23] for first order Hamilton Jacobi equations and then developed by Da Lio and Ley in the case of second order equations. Roughly speaking such a method consists in three main steps: (1) one computes the 
equation satisfied by $w_{\mu}=\mu u-v(u, v$ being respectively the sub and supersolution of the original pde and $0<\mu<1$ a parameter); (2) one constructs a viscosity supersolution of the "linearized" equation $\chi_{\mu}(x, t)$ such that $\chi_{\mu}(x, t) \rightarrow 0$ as $\mu \rightarrow 1$; (3) one shows that $w_{\mu}(x, t) \leq \chi_{\mu}(x, t)$ and then one concludes by letting $\mu \rightarrow 1$. We stress that it is just the condition (0.6) that allows us to build $\chi_{\mu}$. This allows us to construct a strict supersolution to a concave equation associated to $(0.1)$, on which $H_{1}$ remains bounded.

Let us point out that in [18] the coercivity of $H$ with respect to $p \in \mathbb{R}^{N}$, uniformly in $x$ or, in the noncoercive case, the boundedness of $H$ with respect to $x$, uniformly in $p$, allows the authors to take $H_{1}(x, p)=|p|$, $d(x ; 0)=|x|$ and the fact that in this case $d^{2}$ is a $C^{2}$ function plays a key role in the proof of the comparison principle between semicontinuous subsolutions and supersolutions growing at most quadratically in the state variable. Unfortunately such a regularity property is not in general true in this framework. Because of the quite weak conditions on $H$ we are able to get the comparison result in the convex case under conditions on the initial data $h$ and supersolutions $v$ which are stronger than the ones imposed in [18]. Precisely we require that $h, v$ satisfy for some $K>0$

$$
h(x), v(x, t) \geq-K(1+f(d(x))),
$$

where $d(x)=d(x ; 0)$ is the solution of $(0.6)$ and $f: \mathbb{R}^{+} \rightarrow \mathbb{R}^{+}$is a continuous, non decreasing function satisfying

$$
\begin{aligned}
& \lim _{t \rightarrow+\infty} \frac{f(t)}{\Phi^{*}(t)}=0 ; \quad \lim _{t \rightarrow+\infty} \frac{f(C t)}{f(t)}=L_{C}>0 \quad \text { for every constant } C>0 \\
& \limsup _{|x-y| \rightarrow+\infty} \frac{f(d(x ; y))}{|x-y|^{\gamma}}<1 \quad \text { for some } \gamma>1
\end{aligned}
$$

where $\Phi^{*}$ denotes the Legendre-Fenchel transform of $\Phi$ defined in $(\mathbf{H} \mathbf{1})$. It still remains open the case when $f(t)=O\left(\Phi^{*}(t)\right)$ as $t \rightarrow+\infty$, and it will be the aim of future investigation.

By following the same procedure, we can prove a comparison result for the concave problem obtained replacing the Hamiltonian $H$ with $-H$. In this case we should require that the initial data and the subsolutions are bounded from above by $K(1+f(d(x)))$.

In our framework the key hypothesis is the existence of the viscosity solution $d$ of the equation (0.6) (the "intrinsic distance"), which is finite for every $x \in \mathbb{R}^{N}$ and is coercive at infinity so that the closed "balls" associated to $d$ are compact subsets of $\mathbb{R}^{N}$. We note that this hypothesis prescribes in general a growth condition of $H_{1}$, with respect to $x$. Indeed, if we take $H_{1}(x, p)=\left(1+|x|^{2}\right)|p|$, then $d(x ; 0)=\arctan |x|$ does not verifies $(0.7)$. A sufficient condition for $(0.7)$ is that $H_{1}$ grows at most linearly with respect to $x$. Observe that in the case $H_{1}(x, p)=(1+|x|)|p|$, then $d(x ; 0)=\log (1+|x|)$. Nevertheless, some suitable structural conditions on $H_{1}$ could replace the growth condition in order to ensure (0.7). More precisely, if we take $H_{1}(x, p)=\left|\sigma^{T}(x) p\right|$ where $\sigma^{T}$ is the $C^{\infty}$ matrix defining vector fields $X_{i}=\sum_{j=1}^{N} \sigma_{i, j}^{T}(x) \frac{\partial}{\partial x_{j}}$, which are 1-homogeneous with respect to a family of dilation $\delta_{\lambda}$ and left invariant with respect to a group action $\circ$, on a nilpotent stratified Lie group $G=\left(\mathbb{R}^{N}, \circ\right)$, then $\sigma^{T}\left(\delta_{\lambda} x\right)=\lambda \sigma^{T}(x)$ and the linear growth (A1) of $\sigma$ is no more needed. Indeed in this case the solution $d$ of (0.6) is a sub-Riemannian metric of Carnot-Caratheodory type, it is 1-homogeneous w.r.t. $\delta_{\lambda}$ and left invariant with respect to $\circ$. Hence it satisfies $d(x ; y)=d\left(y^{-1} \circ x ; 0\right)$ and $d\left(\delta_{\lambda} x ; 0\right)=\lambda d(x, 0)$, moreover it is finite in the whole space $\mathbb{R}^{N}$ being finite in a neighborhood of $x=0$, in view of the Hörmander condition, guaranteed by the stratification of the group (see e.g. $[26,27]$ or the paper by Gromov in [8]). Thus letting $\lambda \rightarrow \infty$, (choosing $x$ bounded) we immediately get $(0.7)$.

As a consequence of the comparison principle and the Perron's method we get the existence of a continuous solution to the problem (0.1) satisfying the growth condition (0.12).

As far as the issue of the existence is concerned we mention that Capuzzo-Dolcetta and Ishii in [16], see also $[14,15]$, provide the following Hopf-type representation formula for the solution of $(0.1)$

$$
u(x, t)=\inf _{y \in \mathbb{R}^{N}}\left[h(y)+t \Phi^{*}\left(\frac{d(x ; y)}{t}\right)\right],
$$


under the stronger assumption that $(0.6)$ is verified by $H_{0}$ and the initial datum is bounded from below by a function of linear growth with respect to $d(x)=d(x ; 0)$, associated to $H_{0}$. Our result characterizes $(0.14)$ as the unique solution of (0.1) in the set of functions satisfying (0.12).

An analogous uniqueness result for bounded solutions of problem (0.1) with $H_{0}(x, p)=\left|\sigma^{T}(x) p\right|$ where $\sigma^{T}$ is the matrix defining the Heisenberg vector fields has been proved by Manfredi and Stroffolini in [25]. In this paper the authors give a definition of viscosity sub and supersolutions which is linked to the particular structure of the Hamiltonian and thus they prove a uniqueness result in a smaller class of functions. In this regard our result extends the one in [25] since not only it is related to the classical notion of viscosity solution but also it holds in the set of possibly unbounded solutions satisfying (0.12).

We finally mention that recently Birindelli and Wigniolle [10] and Stroffolini [30] studied the homogenization of Hamilton-Jacobi equations respectively on the Heisenberg group and on more general Carnot groups.

The paper is organized as follows. In Section 2 we will premise some basic facts regarding sub-Riemannian metrics and subelliptic operators. In Section 3 we prove a comparison result both in the particular case when the Hamiltonian is quadratic in the gradient and in the more general case when $H$ is given by (0.2). In Section 4 we show the existence of a continuous solution as a consequence of the Comparison Theorem and the Perron's method both in the convex and in the concave case.

\section{Subelliptic metrics AND EIKONAL EQUATIONS}

As we observed in the introduction, the most important hypothesis we make is the existence of a viscosity solution of (0.6) which is finite in the whole space and satisfies (0.7). An important model example where this condition holds true is the case where $H_{1}(x, p)=\left|\sigma^{T}(x) p\right|$ and $\sigma^{T}$ is the matrix associated to a subelliptic operator. For the sake of completeness we recall some basic definitions and important properties of the metrics associated to subelliptic operators. For more details, we refer the reader to $[8,17,20,24,27]$.

We need to introduce some notations. For all integers $N, M \geq 1$ we denote by $\mathcal{M}_{N, M}(\mathbb{R})$ (respectively $\mathcal{S}_{N}(\mathbb{R})$, $\mathcal{S}_{N}^{+}(\mathbb{R})$ ) the set of real $N \times M$ matrices (respectively real symmetric matrices, real symmetric nonnegative $N \times N$ matrices). The standard Euclidean inner product in $\mathbb{R}^{N}$ is written $\langle\cdot, \cdot\rangle$.

Let $A \in C^{2}\left(\mathbb{R}^{N}, \mathcal{S}_{N}^{+}(\mathbb{R})\right)$ and let $L$ denote the operator

$$
L:=\operatorname{div}(A(x) \nabla)
$$

It is a well-known fact that the symmetric matrix $A$, can be factorized as $A(x)=\sigma(x) \sigma^{T}(x)$ with $\sigma \in$ $C_{\text {loc }}^{0,1}\left(\mathbb{R}^{N}, \mathcal{M}_{N, M}(\mathbb{R})\right)$. Thus the operator $L$ can be written as

$$
L=\sum_{i=1}^{M} X_{i}^{*} X_{i}
$$

with $X_{i}=\sum_{j=1}^{N} \sigma_{i j}^{T} \frac{\partial}{\partial x_{j}}$ and $X_{i}^{*}$ denotes the formal adjoint of $X_{i}$ in $L^{2}$.

As in the case of the uniformly elliptic operators, it is possible to associate to $L$ an intrinsic distance, denoted here by $d$, defined as the minimal time function for a suitable control system.

We recall that $v \in \mathbb{R}^{N}$ is a subunit vector with respect to $L$ at the point $x$ if

$$
|v \cdot \eta|^{2} \leq A(x) \eta \cdot \eta, \forall \eta \in \mathbb{R}^{N}
$$

We observe that since $A(x)=\sigma(x) \sigma^{T}(x),(1.2)$ is equivalent to

$$
|v \cdot \eta| \leq\left|\sigma^{T}(x) \eta\right|, \forall \eta \in \mathbb{R}^{N} .
$$

Moreover the set of subunit vectors at a point $x$ w.r.t. $L$ is a convex set. 
The intrinsic distance $d$ associated with $L$ is defined as follows

Definition 1.1. For every $x, y \in \mathbb{R}^{N}$

$$
\begin{array}{r}
d(x ; y)=\inf \left\{T>0: \exists \gamma:[0, \infty) \rightarrow \mathbb{R}^{N} \text { Lipschitz continuous such that } \gamma(0)=x,\right. \\
\gamma(T)=y \text { and } \dot{\gamma}(t) \text { is subunit at } \gamma(t) \quad \text { for a.e. } t \in(0, T)\}
\end{array}
$$

In the case $L=\Delta$ the intrinsic distance coincides with the Euclidean distance and more generally if $L$ is uniformly elliptic, $d$ provides a distance which is equivalent to the Euclidean one.

The following characterization of subunit vectors allows to consider $d(x ; y)$ as the minimal time function for a control system associated with $L$.

Proposition 1.1. A vector $v \in \mathbb{R}^{N}$ is a subunit vector at $x \in \mathbb{R}^{N}$ with respect to the operator $L$ if and only if there exists $w \in \mathbb{R}^{M}$ with $|w| \leq 1$ such that $v=\sigma(x) w$.

Proof. We give the proof of the "only if" part just for the reader's convenience. Let us denote by $\mathcal{B}_{x}$ the set of subunit vectors at $x \in \mathbb{R}^{N}$ with respect to the operator $L$ and by $\mathcal{C}_{x}:=\left\{\sigma(x) w: w \in \mathbb{R}^{M},|w| \leq 1\right\}$. We observe that $\mathcal{C}_{x}$ is a closed and convex subset of $\mathbb{R}^{N}$. We suppose by contradiction that there is $v \in \mathcal{B}_{x} \backslash \mathcal{C}_{x}$. By Hahn-Banach Theorem there are $b \in \mathbb{R}^{N}$ and $\alpha>0$ such that $\langle v, b\rangle \geq \alpha$ and $\langle\sigma(x) w, b\rangle\langle\alpha$ for all $|w| \leq 1$. Let us take $w=\frac{\sigma^{T}(x) b}{\left|\sigma^{T}(x) b\right|}$. With this choice of $w$ we have the following estimate

$$
\alpha \leq|\langle v, b\rangle| \leq\left|\sigma^{T}(x) b\right|=\left\langle\sigma^{T}(x) w, b\right\rangle<\alpha,
$$

which is a contradiction and we conclude.

The previous proposition allows to define $d(x ; y)$ as the minimal time function associated to the following symmetric control system:

$$
\left\{\begin{array}{l}
\dot{y}(t, u)=\sigma(y(t, u)) u(t), \quad u(t) \in \mathbb{R}^{M},|u(t)| \leq 1 \\
y(0, u)=x
\end{array}\right.
$$

where the control $u \in \mathcal{U}:=\left\{u:[0, \infty) \rightarrow \mathbb{R}^{M}, u\right.$ measurable $\}$ and the target $\mathcal{T}=\{y\}$. Indeed, the Dynamic Programming Principle (see e.g. $[3,5]$ ) allows to prove that $d_{y}(x)=d(x ; y)$ satisfies, in the viscosity sense, the equation (0.6), with

$$
H_{1}(x, p)=\sup _{|u| \leq 1}\{-\sigma(x) u \cdot p\}=\sup _{|u| \leq 1}\left\{-u \cdot \sigma^{T}(x) p\right\}
$$

Hence, being the sup attained at $u=-\frac{\sigma^{T}(x) p}{\left|\sigma^{T}(x) p\right|}$ if $\sigma^{T}(x) p \neq 0$ and at $u=0$ in the other case, we get $H_{1}(x, p)=$ $\left|\sigma^{T}(x) p\right|$.

Let us mention that the symmetric control systems (1.6) play an important role for robotic applications to wheeled vehicles, to object manipulation and for the study of nonholonomic systems (see e.g. [12]).

Assume $L$ satisfies the following hypothesis:

(A2) $\mid \begin{aligned} & \text { there exists } m \in \mathbb{N} \text { such that the Lie algebra spanned by the vect } \\ & X_{i} \text { for } i=1, \ldots, M \text { by means of commutators of length } \leq m \text { has }\end{aligned}$ $\operatorname{rank} N$ at all points $x \in \mathbb{R}^{N}$.

Observe that the hypothesis (A2) is equivalent to the subelliptic estimate

$$
\|u\|_{H^{\epsilon}} \leq\|u\|_{L^{2}}+\|L u\|_{L^{2}}
$$

with $\epsilon=\frac{1}{m}$ (see [21]) and therefore it characterizes the subelliptic operators. 
In [20] Fefferman and Phong proved that (1.7) is equivalent to

$$
\exists C>0 \text { such that } B(x, R) \subseteq B_{L}\left(x, C R^{\frac{1}{m}}\right) \subseteq B\left(x, R^{\frac{1}{m}}\right) \forall R<R_{0} ; \forall x \in \mathbb{R}^{N}
$$

where $B_{L}$ denotes the ball associated to the intrinsic distance $d$, and $B$ denotes the euclidean ball. Thus the topology induced by $d$ is equivalent to the euclidean topology. We observe that the condition (1.8) can be considered as a controllability property for the control system (1.6). Indeed, it ensures that the reachable set at time $t$,

$$
\mathcal{R}(t)=\left\{z \in \mathbb{R}^{N}: \text { there exists } u \in \mathcal{U} \text { such that } y(0, u)=z \text { and } y(\tau, u)=y \text { for some } \tau \leq t\right\}
$$

contains an euclidean ball, for all $t>0$ small enough. This property is known in the literature as small time local controllability (in short STLC) property (see [31]).

Finally let us assume that the matrix $\sigma(x)$ which defines the operator $L$ satisfies the additional assumption $(A 1)$. Then the following result holds (see e.g. $[3,16]$ ).

Theorem 1.1. Assume that $\sigma \in C_{\text {loc }}^{0,1}\left(\mathbb{R}^{N}, \mathcal{M}_{N, M}(\mathbb{R})\right)$ and satisfies (A1) and (A2). Then $d(x ; y)$ is the unique nonnegative viscosity solution of the problem $(0.6)$, with $H_{1}(x, p)=\left|\sigma^{T}(x) p\right|$. Moreover, it satisfies $d(x ; y)<+\infty$ for every $x, y \in \mathbb{R}^{N}, \lim _{|x-y| \rightarrow+\infty} d(x ; y)=+\infty$, the inequality $(0.8)$, and the local Hölder continuity (0.9) of exponent $\alpha=\frac{1}{m}$ with $m$ defined in (A2).

Remark 1.1. The hypothesis (A1), which is relative to the behavior of $\sigma$ for $|x|$ large, ensures in particular the existence and uniqueness of a global solution of (1.6) for fixed $u \in \mathcal{U}$. In particular (A2) holds true if $x \rightarrow \sigma(x)$ is Lipschitz continuous in $\mathbb{R}^{N}$, which is the case if the matrix $A(x)=\sigma(x) \sigma^{T}(x)$ satisfies $A_{i j} \in C^{2}\left(\mathbb{R}^{N}, \mathbb{R}\right)$ with $\left|\nabla^{2} A_{i j}(x)\right| \leq M$ for a suitable $M>0$.

In the framework of the subelliptic operators, the sublaplacians on nilpotent stratified Lie groups play a fundamental role. These operators are left invariant with respect to the action $\circ$ of a group and are homogeneous with respect to a family of dilations in a nilpotent stratified Lie group $G$.

In this case (1.8) holds true with $R_{0}=+\infty$ and the distance $d(x ; 0)$ is homogeneous of degree one with respect to an intrinsic dilation $\delta_{\lambda}$ of the group, namely it satisfies

$$
d\left(\delta_{\lambda}(y) ; 0\right)=\lambda d(y ; 0) .
$$

Moreover, $d(x ; y)$ can be defined through the group law $\circ$ by putting $d(x, y)=d\left(y^{-1} \circ x, 0\right)$ where $y^{-1}$ is the inverse of $y$ with respect to $\circ$, i.e. $y^{-1}=-y$.

Thus $d(x ; y)$ is finite for every $x, y \in G$ and it goes to infinity as $|x-y| \rightarrow+\infty$. For this reason, in this particular case, hypothesis (A1) is no more needed to ensure the previous properties. Furthermore, in this case, $d(x ; y) \leq C|x-y|$ as $|x-y| \rightarrow \infty$.

The most important example of sublaplacian on a nilpotent stratified Lie group is the Heisenberg Laplacian described in the following example.

Example 1. Take the Heisenberg group $G=H^{n}=\left(\mathbb{R}^{2 n+1}, \circ\right)$ where the group action $\circ$ is defined as

$$
x \circ y=\left(x_{1}+y_{1}, \ldots, x_{2 n}+y_{2 n}, x_{2 n+1}+y_{2 n+1}+2 \sum_{i=1}^{n}\left(x_{n+i} y_{i}-x_{i} y_{n+i}\right)\right),
$$

and the dilation is given by

$$
\delta_{\lambda}(y)=\left(\lambda y_{1}, \ldots, \lambda y_{2 n}, \lambda^{2} y_{2 n+1}\right) .
$$

The fields $X_{i}$ defining the Heisenberg Laplacian are given in this case by

$$
X_{i}=\frac{\partial}{\partial x_{i}}+2 x_{i+n} \frac{\partial}{\partial x_{2 n+1}}, \quad X_{i+n}=\frac{\partial}{\partial x_{i+n}}-2 x_{i} \frac{\partial}{\partial x_{2 n+1}}
$$


for $i=1, \ldots, n$. Moreover, the eikonal equation (0.6) is

$$
H_{1}(x, p)=\left(\sum_{i=1}^{n}\left(p_{i}+2 x_{i+n} p_{2 n+1}\right)^{2}+\left(p_{i+n}-2 x_{i} p_{2 n+1}\right)^{2}\right)^{\frac{1}{2}}=1 .
$$

Let us mention that $H_{1}$ in this case is the Hamiltonian associated, by the Dynamic Programming Principle, to the famous Brockett system (1.6), which can be viewed as the prototype for first bracket controllable systems involved to model nonholonomic effects, see $[11,12]$.

\section{Comparison Result}

The aim of this section is to prove a comparison result between unbounded semicontinuous viscosity subsolutions and supersolutions of the following Cauchy problem

$$
\begin{cases}u_{t}+H(x, D u)=0 & \text { in } \mathbb{R}^{N} \times(0, T) \\ u(x, 0)=h(x) & \text { in } \mathbb{R}^{N}\end{cases}
$$

We assume that the Hamiltonian $H: \mathbb{R}^{2 N} \rightarrow \mathbb{R}$ satisfies (H1) and (H2).

We will start by considering the model case (0.10) and then the more general case.

Next we recall a known result for convex Hamiltonians that we will use in the sequel (see e.g. $[3,5]$ ).

Theorem 2.1. Let $\Omega \subseteq \mathbb{R}^{N}$ be an open set and let $F: \bar{\Omega} \times \mathbb{R} \times \mathbb{R}^{N} \rightarrow \mathbb{R}$ be a continuous function such that

$$
p \rightarrow F(x, r, p) \quad \text { is convex }
$$

and

$$
|F(x, r, p)-F(y, r, p)| \leq m_{R}(|x-y|(1+|p|))
$$

for all $|x|,|y| \leq R, p \in \mathbb{R}^{N}$ and $|r| \leq R$ and for some modulus of continuity $m_{R}$. Then $u \in C\left(\mathbb{R}^{N}\right)$ is a viscosity solution of the first order equation

if and only if

$$
F(x, u, D u)=0 \text { in } \Omega
$$

$$
F(x, u(x), p)=0, \quad \text { for all } x \in \Omega, p \in D^{-} u(x)
$$

If $\sigma$ is a matrix satisfying the hypotheses of Theorem 1.1 then Theorems 1.1 and 2.1 yield that for every $x_{0} \in \mathbb{R}^{N} \backslash\{0\}$ and $p \in D^{-} d\left(x_{0}\right)$

$$
\left|\sigma^{T}\left(x_{0}\right) p\right|=1
$$

Given $T>0$, we denote by $U S C\left(\mathbb{R}^{N} \times[0, T]\right)$ the set of upper semicontinuous functions in $\mathbb{R}^{N} \times[0, T]$ and by $L S C\left(\mathbb{R}^{N} \times[0, T]\right)$ the set of lower semicontinuous functions in $\mathbb{R}^{N} \times[0, T]$.

\subsection{The quadratic case}

In this section we are going to prove the Comparison Principle in the particular case when the Hamiltonian is given by (0.10) with $\beta=2$ and $b$ satisfying (0.11). On the initial data $h$ and the supersolution $v$, we assume that for some $K>0$,

$$
h(x), v(x, t) \geq-K(1+d(x))
$$

where $d(x)=d(x ; 0)$ is defined in $(0.6)$ with $H_{1}(x, p)=\left|\sigma^{T}(x) p\right|$. This corresponds to the case $f \equiv I$ in $(0.12)$ and (0.13). Moreover we suppose that (0.13) is verified with $\gamma=2$, namely

$$
\limsup _{|x-y| \rightarrow+\infty} \frac{d(x ; y)}{|x-y|^{2}}<1 \text {. }
$$


The extension of the result to more general Hamiltonians and growth conditions on the solutions and initial data will be provided in the next section.

More precisely we consider the following Cauchy problem

$$
\begin{cases}u_{t}(x, t)+\frac{1}{2}\left|b^{T}(x) D u\right|^{2}=0 & \text { in } \mathbb{R}^{N} \times(0, T) \\ u(x, 0)=h(x) & \text { in } \mathbb{R}^{N}\end{cases}
$$

under the following basic hypotheses on the data:

(Q1) (Assumption on $b): b \in C\left(\mathbb{R}^{N} ; \mathcal{M}_{N, M}(\mathbb{R})\right.$ ) is locally Lipschitz continuous and verifies

$$
C \sigma(x) \sigma^{T}(x)-b(x) b^{T}(x) \geq 0 \text { for all } x \in \mathbb{R}^{N},
$$

for some constant $C>0$ and for some matrix $\sigma$ satisfying the hypotheses of Theorem 1.1.

(Q2) (Assumption on the initial condition $h): h \in C\left(\mathbb{R}^{N} ; \mathbb{R}\right)$ and $h(x) \geq-K(1+d(x)$ ) for some $K>0$, where $d(x)=d(x ; 0)$ is defined in $(0.6)$.

Theorem 2.2. Assume (Q1)-(Q2). Let $u \in U S C\left(\mathbb{R}^{N} \times[0, T]\right)$ and $v \in L S C\left(\mathbb{R}^{N} \times[0, T]\right)$ be respectively a viscosity subsolution and supersolution of the problem (2.6). Assume that for some $K>0$ we have $v(x, t) \geq$ $-K(1+d(x))$. Then $u(x, t) \leq v(x, t)$ in $\mathbb{R}^{N} \times[0, T]$.

Before proving the theorem, let us observe that without loss of generality, we may suppose that $u$ is bounded from above, otherwise we replace $u$ with $\tilde{u}(x, t)=g(u(x, t))$ where $g$ is a $C^{1}$ approximation of the function

$$
g_{\gamma}(r)=r \wedge \gamma
$$

satisfying $g(0)=0$ and $0<g^{\prime}(x) \leq 1$. Indeed, $\tilde{u}$ satisfies

$$
\tilde{u}_{t}+\frac{1}{2}\left|b^{T}(x) D \tilde{u}\right|^{2} \leq g^{\prime}(u)\left[u_{t}+\frac{1}{2}\left|b^{T}(x) D u\right|^{2}\right] \leq 0
$$

and $\tilde{u}(x, 0)=g(u(x, 0)) \leq g(h(x)) \leq h(x)$. Hence, $\tilde{u}$ is a subsolution of $(2.6)$ and if we prove that

$$
g_{\gamma}(u) \leq v \quad \forall \gamma
$$

we obtain the result by letting $\gamma \rightarrow+\infty$.

Hence we will prove the theorem by assuming that $u$ is bounded from above.

Proof of Theorem 2.2. We divide the proof in several steps by following the strategy of proof of Theorem 1.1 in [18].

Step 1. Let $\mu \in(0,1)$ and set $w(x, t)=\mu u(x, t)-v(x, t)$. Then $w$ is an USC viscosity subsolution of

$$
\begin{cases}w_{t}(x, t)-\frac{1}{2(1-\mu)}\left|b^{T}(x) D w(x, t)\right|^{2}=0 & \text { in } \mathbb{R}^{N} \times(0, T) \\ w(x, 0)=(1-\mu) K(1+d(x)) & \text { in } \mathbb{R}^{N} .\end{cases}
$$

Indeed we have

$$
w(x, 0)=\mu u(x, 0)-v(x, 0) \leq(\mu-1) h(x) \leq(1-\mu) K(1+d(x)) .
$$

Now let $\phi \in C^{1}\left(\mathbb{R}^{N} \times[0, T]\right)$ and $(\bar{x}, \bar{t}) \in \mathbb{R}^{N} \times(0, T]$ be a strict maximum of $w-\phi$ in $\bar{B}(\bar{x}, r) \times[\bar{t}-r, \bar{t}+r]$ for some $r>0$. For all $\varepsilon>0$ we set

$$
\psi_{\varepsilon}(x, y, t)=\phi(x, t)+\frac{|x-y|^{2}}{\varepsilon^{2}}
$$


and consider

$$
M_{\varepsilon}:=\max _{x, y \in \bar{B}(\bar{x}, r), t \in[\bar{t}-r, \bar{t}+r]}\left\{\mu u(x, t)-v(y, t)-\psi_{\varepsilon}(x, y, t)\right\} .
$$

This maximum is achieved at a point $\left(x_{\varepsilon}, y_{\varepsilon}, t_{\varepsilon}\right)$ and, since $(\bar{x}, \bar{t})$ is a strict maximum of $w-\phi$, standard arguments show that as $\varepsilon \rightarrow 0$, we have

$$
\frac{\left|x_{\varepsilon}-y_{\varepsilon}\right|^{2}}{\varepsilon^{2}} \rightarrow 0
$$

and

$$
M_{\varepsilon}=\mu u\left(x_{\varepsilon}, t_{\varepsilon}\right)-v\left(y_{\varepsilon}, t_{\varepsilon}\right)-\psi_{\varepsilon}\left(x_{\varepsilon}, y_{\varepsilon}, t_{\varepsilon}\right) \longrightarrow \mu u(\bar{x}, \bar{t})-v(\bar{x}, \bar{t})-\phi(\bar{x}, \bar{t})=w(\bar{x}, \bar{t})-\phi(\bar{x}, \bar{t}) .
$$

It means that, at the limit $\varepsilon \rightarrow 0$, we obtain some information on $w-\phi$ at $(\bar{x}, \bar{t})$ which will provide the new equation for $w$. Before that we can take $\psi_{\varepsilon}$ as a test function to use the fact that $\mu u$ is a subsolution and $v$ is a supersolution. Indeed $(x, t) \in \bar{B}(\bar{x}, r) \times[\bar{t}-r, \bar{t}+r] \mapsto \mu u(x, t)-v\left(y_{\varepsilon}, t\right)-\psi_{\varepsilon}\left(x, y_{\varepsilon}, t\right)$ achieves its maximum at $\left(x_{\varepsilon}, t_{\varepsilon}\right)$ and $(y, t) \in \bar{B}(\bar{x}, r) \times[\bar{t}-r, \bar{t}+r] \mapsto-\mu u\left(x_{\varepsilon}, t\right)+v(y, t)+\psi_{\varepsilon}\left(x_{\varepsilon}, y, t\right)$ achieves its minimum at $\left(y_{\varepsilon}, t_{\varepsilon}\right)$. Thus there exist $a_{1}, a_{2} \in \mathbb{R}$ such that if $p_{\varepsilon}=\frac{2\left(x_{\varepsilon}-y_{\varepsilon}\right)}{\varepsilon^{2}}$ we have

$$
\begin{gathered}
\left(a_{1}, p_{\varepsilon}+D_{x} \phi_{\varepsilon}\left(x_{\varepsilon}, y_{\varepsilon}, t_{\varepsilon}\right)\right) \in D^{+} \mu u\left(x_{\varepsilon}, t_{\varepsilon}\right) \\
\left(a_{2}, p_{\varepsilon}\right) \in D^{-} v\left(y_{\varepsilon}, t_{\varepsilon}\right) ; \\
a_{1}-a_{2}=\frac{\partial}{\partial t} \psi_{\varepsilon}\left(x_{\varepsilon}, y_{\varepsilon}, t_{\varepsilon}\right)=\frac{\partial}{\partial t} \phi\left(x_{\varepsilon}, t_{\varepsilon}\right) ; \\
a_{1}+\frac{1}{2 \mu}\left|b^{T}\left(x_{\varepsilon}\right) p_{\varepsilon}+b^{T}\left(x_{\varepsilon}\right) D_{x} \phi\left(x_{\varepsilon}, t_{\varepsilon}\right)\right|^{2} \leq 0
\end{gathered}
$$

and

$$
a_{2}+\frac{1}{2}\left|b^{T}\left(y_{\varepsilon}\right) p_{\varepsilon}\right|^{2} \geq 0
$$

By subtracting (2.12) to (2.11) we get

$$
a_{1}-a_{2}+\frac{1}{2 \mu}\left|b^{T}\left(x_{\varepsilon}\right) p_{\varepsilon}+b^{T}\left(x_{\varepsilon}\right) D_{x} \phi\left(x_{\varepsilon}, t_{\varepsilon}\right)\right|^{2}-\frac{1}{2}\left|b^{T}\left(y_{\varepsilon}\right) p_{\varepsilon}\right|^{2} \leq 0 .
$$

The convexity of $p \rightarrow|p|^{2}$ implies the following inequality

$$
\mu|p|^{2}-|\mu p-q|^{2} \geq-\frac{1}{1-\mu}|q|^{2} \quad \text { for } \mu \in(0,1) .
$$

Using the above inequality with

$$
p=b^{T}\left(x_{\varepsilon}\right) \frac{p_{\varepsilon}}{\mu}+b^{T}\left(x_{\varepsilon}\right) \frac{D_{x} \phi\left(x_{\varepsilon}, t_{\varepsilon}\right)}{\mu} \quad \text { and } q=\left(b^{T}\left(x_{\varepsilon}\right)-b^{T}\left(y_{\varepsilon}\right)\right) p_{\varepsilon}+b^{T}\left(x_{\varepsilon}\right) D_{x} \phi\left(x_{\varepsilon}, t_{\varepsilon}\right)
$$

and taking into account that for some $L>0,\left|\left(b^{T}\left(x_{\varepsilon}\right)-b^{T}\left(y_{\varepsilon}\right)\right) p_{\varepsilon}\right| \leq L \frac{\left|x_{\varepsilon}-y_{\varepsilon}\right|^{2}}{\varepsilon^{2}}=o_{\varepsilon}(1)$ as $\varepsilon \rightarrow 0(b$ being locally Lipschitz) we get

$$
\begin{gathered}
0 \geq \phi_{t}\left(x_{\varepsilon}, t_{\varepsilon}\right)-\frac{1}{2(1-\mu)}\left|b^{T}\left(x_{\varepsilon}\right) D_{x} \phi\left(x_{\varepsilon}, t_{\varepsilon}\right)+\left(b^{T}\left(x_{\varepsilon}\right)-b^{T}\left(y_{\varepsilon}\right)\right) p_{\varepsilon}\right|^{2} \\
=\phi_{t}\left(x_{\varepsilon}, t_{\varepsilon}\right)-\frac{1}{2(1-\mu)}\left|b^{T}\left(x_{\varepsilon}\right) D_{x} \phi\left(x_{\varepsilon}, t_{\varepsilon}\right)+o_{\varepsilon}(1)\right|^{2}
\end{gathered}
$$

Now by letting $\varepsilon \rightarrow 0$ we obtain that $w$ is a viscosity subsolution of $(2.7)$. 
On the other hand (0.11) implies that

$$
\left|b^{T}(x) p\right|^{2} \leq C\left|\sigma^{T}(x) p\right|^{2}
$$

for every $p \in \mathbb{R}^{N}$. Thus, $w$ is a viscosity subsolution also of

$$
\begin{cases}w_{t}(x, t)-\frac{1}{2(1-\mu)}\left|\sigma^{T}(x) D w(x, t)\right|^{2}=0 & \text { in } \mathbb{R}^{N} \times(0, T) \\ w(x, 0)=(1-\mu) K(1+d(x)) & \text { in } \mathbb{R}^{N}\end{cases}
$$

Step 2. Now we are going to construct a viscosity supersolution of the equation $(2.14)$ in $\mathbb{R}^{N} \times[0, \tau]$ for some $\tau \in(0, T)$ and then we proceed by using a bootstrap argument on the parameter $\tau$. To this purpose let us set for $\delta \in(0,1)$ and $K^{\prime}>2 K$,

$$
\chi_{\delta}(x, t)=(1-\mu) K^{\prime} \frac{1+\left[(d(x)-\delta)^{+}\right]^{2}}{1-4 M t}
$$

where $M>0$ will be determined later and $d(x)=d(x ; 0)$ is the solution of $(0.6)$ with $H_{1}(x, p)=\left|\sigma^{T}(x) p\right|$.

Lemma 2.1. The function defined in (2.15) is a viscosity supersolution of $(2.14)$ in $\mathbb{R}^{N} \times[0, \tau]$ for some $0<\tau<\frac{1}{4 M}$.

Proof of Lemma 2.1. We first observe that, being $K^{\prime}>2 K$, we have

$$
\chi_{\delta}(x, 0) \geq(1-\mu) K(1+d(x)) .
$$

Moreover the following estimates hold

$$
\frac{\partial}{\partial t} \chi_{\delta}(x, t)=(1-\mu) 4 M K^{\prime} \frac{1+\left[(d(x)-\delta)^{+}\right]^{2}}{(1-4 M t)^{2}}
$$

and for every $p \in D^{-} \chi_{\delta}(x, t)$ there is $q \in D^{-} d(x)$ such that

$$
\left|\sigma^{T}(x) p\right|=\frac{2 K^{\prime}(1-\mu)(d(x)-\delta)^{+}}{(1-4 M t)}\left|\sigma^{T}(x) q\right|
$$

By Theorem 2.1 we have $\left|\sigma^{T}(x) q\right|=1$ for all $q \in D^{-} d(x)$. Thus for all $p \in D^{-} \chi_{\delta}(x, t)$ and for all $t<\frac{1}{4 M}$ we have

$$
\frac{\partial}{\partial t} \chi_{\delta}(x, t)-\frac{1}{2(1-\mu)}\left|\sigma^{T}(x) p\right|^{2} \geq(1-\mu)\left[\frac{4 M K^{\prime}}{(1-4 M t)^{2}}+2 K^{\prime}\left(2 M-K^{\prime}\right) \frac{\left[(d(x)-\delta)^{+}\right]^{2}}{(1-4 M t)^{2}}\right] .
$$

Hence, if we choose $M>\frac{K^{\prime}}{2}$ and $\tau<\frac{1}{4 M}$, then for all $t \in(0, \tau]$ the last term in the previous inequality is strictly bigger than zero. Therefore $\chi_{\delta}$ is a viscosity supersolution of the equation (2.14) and we can conclude.

We continue the proof of Theorem 2.2. We notice that for fixed $\delta>0, \chi_{\delta}=O\left(d^{2}(x)\right)$ as $|x| \rightarrow+\infty$. Thus we have

$$
\lim _{|x| \rightarrow+\infty} w(x, t)-\chi_{\delta}(x, t)=-\infty
$$

Hence, $M=\max _{\mathbb{R}^{N} \times[0, \tau]}\left[w(x, t)-\chi_{\delta}(x, t)\right]$ is attained at a point $\left(x_{0}, t_{0}\right)$ with $d\left(x_{0}\right) \leq R_{0}$, for some $R_{0}>0$. We assume by contradiction that $M>0$ and $t_{0}>0$. Then for $\eta>0$ small enough we still have $M_{\eta}=$ $\max _{\left.\mathbb{R}^{N} \times[0, \tau]\right)}\left[w(x, t)-\chi_{\delta}(x, t)-\eta t\right]>0$. 
For all $\varepsilon>0$ we introduce the auxiliary function

$$
\Phi_{\varepsilon, \eta}(x, y, t)=w(x, t)-\chi_{\delta}(y, t)-\eta t-\frac{|x-y|^{2}}{2 \varepsilon}
$$

We claim that the $\max _{\mathbb{R}^{N} \times \mathbb{R}^{N} \times[0, \tau]} \Phi_{\varepsilon, \eta}(x, y, t)$ is achieved at a point $\left(x_{\varepsilon}, y_{\varepsilon}, t_{\varepsilon}\right)$, lying in a compact set independent of $\varepsilon$. Indeed by using the triangular inequality (0.8) satisfied by $d$, for some $C>0$ and for $\varepsilon$ small we get

$$
\begin{aligned}
\Phi_{\varepsilon, \eta}(x, y, t) & \leq C\left(1+d(x)-d^{2}(y)-|x-y|^{2}\right) \\
& \leq C\left(1+d(x ; y)+d(y)-d^{2}(y)-|x-y|^{2}\right) .
\end{aligned}
$$

If $|x-y| \rightarrow+\infty$, as $|x|,|y| \rightarrow+\infty$, then because of (2.5) we have $d(x ; y)-|x-y|^{2} \rightarrow-\infty$. On the other hand if $|x-y| \rightarrow \ell<+\infty$ as $|x|,|y| \rightarrow+\infty$, then $d(x ; y)-|x-y|^{2}$ remains bounded since $d$ is locally Hölder continuous in $\mathbb{R}^{N}$. Thus

$$
\limsup _{|x|,|y| \rightarrow+\infty} \Phi_{\varepsilon, \eta}(x, y, t)=-\infty
$$

and we prove the claim.

Moreover standard arguments show that (up to subsequences) as $\varepsilon \rightarrow 0$

$$
\begin{aligned}
& \frac{\left|x_{\varepsilon}-y_{\varepsilon}\right|^{2}}{2 \varepsilon} \rightarrow 0, \quad t_{\varepsilon} \rightarrow \bar{t}>0 \\
& \Phi_{\varepsilon}\left(x_{\varepsilon}, y_{\varepsilon}, t_{\varepsilon}\right) \rightarrow M_{\eta} .
\end{aligned}
$$

Thus there are $a_{1}, a_{2} \in \mathbb{R}$ such that such that if $p_{\varepsilon}=\frac{\left(x_{\varepsilon}-y_{\varepsilon}\right)}{\varepsilon}$

$$
\begin{gathered}
\left(a_{1}, p_{\varepsilon}\right) \in D^{+} w\left(x_{\varepsilon}, t_{\varepsilon}\right) ; \\
\left(a_{2}, p_{\varepsilon}\right) \in D^{-} \chi_{\delta}\left(y_{\varepsilon}, t_{\varepsilon}\right) ; \\
a_{1}-a_{2}=\eta ; \\
\left.a_{1}-\frac{1}{2(1-\mu)} \mid \sigma^{T}\left(x_{\varepsilon}\right) p_{\varepsilon}\right)\left.\right|^{2} \leq 0
\end{gathered}
$$

and

$$
a_{2}-\frac{1}{2(1-\mu)}\left|\sigma^{T}\left(y_{\varepsilon}\right) p_{\varepsilon}\right|^{2} \geq 0 .
$$

By subtracting (2.20) to (2.19) we get

$$
\left.\eta-\frac{1}{2(1-\mu)} \mid \sigma^{T}\left(x_{\varepsilon}\right) p_{\varepsilon}\right)\left.\right|^{2}+\frac{1}{2(1-\mu)}\left|\sigma^{T}\left(y_{\varepsilon}\right) p_{\varepsilon}\right|^{2} \leq 0
$$

Now we recall that for every matrix $B(x)$ and every vector $\xi \in \mathbb{R}^{N}$,we have

$$
|B(x) \xi|^{2}-|B(y) \xi|^{2}=\langle(B(x)-B(y)) \xi,(B(x)+B(y)) \xi\rangle .
$$

Moreover from (2.16) we have $\left|\sigma^{T}\left(y_{\varepsilon}\right) p_{\varepsilon}\right| \leq \frac{2 K^{\prime}(1-\mu)\left[d\left(y_{\varepsilon}\right)-\delta\right]^{+}}{(1-4 M t)}$. 
Thus since $\sigma$ is locally Lipschitz we find

$$
\begin{aligned}
0<\eta & <\frac{1}{2(1-\mu)}\left\langle\left(\sigma^{T}\left(x_{\varepsilon}\right)-\sigma^{T}\left(y_{\varepsilon}\right)\right)\left(\frac{x_{\varepsilon}-y_{\varepsilon}}{\varepsilon}\right),\left(\sigma^{T}\left(x_{\varepsilon}\right)+\sigma^{T}\left(y_{\varepsilon}\right)\right)\left(\frac{x_{\varepsilon}-y_{\varepsilon}}{\varepsilon}\right)\right\rangle \\
& \leq \frac{1}{2(1-\mu)}\left|\sigma^{T}\left(x_{\varepsilon}\right)-\sigma^{T}\left(y_{\varepsilon}\right)\right| \frac{x_{\varepsilon}-y_{\varepsilon}}{\varepsilon}||\left(\sigma^{T}\left(x_{\varepsilon}\right)+\sigma^{T}\left(y_{\varepsilon}\right)\right)\left(\frac{x_{\varepsilon}-y_{\varepsilon}}{\varepsilon}\right) \mid \\
& \leq \frac{1}{2(1-\mu)} L \frac{\left|x_{\varepsilon}-y_{\varepsilon}\right|^{2}}{\varepsilon}\left[\left|\sigma^{T}\left(y_{\varepsilon}\right) \frac{\left(x_{\varepsilon}-y_{\varepsilon}\right)}{\varepsilon}\right|+\left|\left(\sigma^{T}\left(x_{\varepsilon}\right)-\sigma^{T}\left(y_{\varepsilon}\right)+\sigma^{T}\left(y_{\varepsilon}\right)\right) \frac{\left(x_{\varepsilon}-y_{\varepsilon}\right)}{\varepsilon}\right|\right] \\
& \leq \frac{1}{2(1-\mu)} L \frac{\left|x_{\varepsilon}-y_{\varepsilon}\right|^{2}}{\varepsilon}\left(L \frac{\left|x_{\varepsilon}-y_{\varepsilon}\right|^{2}}{\varepsilon}+2 \frac{2 K^{\prime}(1-\mu)\left[d\left(y_{\varepsilon}\right)-\delta\right]^{+}}{(1-4 M \tau)}\right) .
\end{aligned}
$$

Now letting $\varepsilon \rightarrow 0$ we get $0<\eta \leq 0$ which is a contradiction. Therefore, either the maximum $M$ of $w(x, t)-$ $\chi_{\delta}(x, t)$ vanishes or it is achieved at time $t_{0}=0$. Since by construction we have $w\left(x_{0}, 0\right)-\chi_{\delta}\left(x_{0}, 0\right) \leq 0$ then for all $(x, t) \in \mathbb{R}^{N} \times[0, \tau]$ we obtain $w(x, t)-\chi_{\delta}(x, t) \leq 0$. Thus

$$
w(x, t) \leq(1-\mu) K^{\prime} \frac{1+\left[(d(x)-\delta)^{+}\right]^{2}}{1-4 M t} .
$$

Finally letting $\mu \rightarrow 1$ we get the claim $u(x, t) \leq v(x, t)$ and we conclude by a boostrap argument.

\subsection{The general case}

In this section we prove the comparison result to the more general Cauchy problem (0.1) where the Hamiltonian $H$ satisfies the hypotheses (H1) and (H2).

Let $f: \mathbb{R}^{+} \rightarrow \mathbb{R}^{+}$be a continuous function satisfying (0.12) and (0.13).

We will use the following assumption on $h$.

(Q2bis) (Assumption on the initial condition $h): h \in C\left(\mathbb{R}^{N}\right)$ and $h(x) \geq-K(1+f(d(x)))$ for some $K>0$, where $d(x)=d(x ; 0)$ is defined in $(0.6)$.

Theorem 2.3. Assume (H1), (H2) and (Q2bis). Let $u \in U S C\left(\mathbb{R}^{N} \times[0, T)\right)$ and $v \in L S C\left(\mathbb{R}^{N} \times[0, T)\right)$ be respectively a viscosity subsolution and supersolution to the problem (0.1). Assume that $v(x, t) \geq-K(1+$ $f(d(x)))$. Then $u(x, t) \leq v(x, t)$ in $\mathbb{R}^{N} \times[0, T]$.

Proof. The strategy of proof is the same of that of Theorem 2.2 and we will reproduce only the key points. As in Theorem 2.2 we may assume without loss of generality that $u$ is bounded from above. We first suppose $\Phi^{*}$ is a $C^{1,1}$ strictly increasing function in $\mathbb{R}$ and then we show how to reduce to this case by using standard approximation arguments.

Step 1. Suppose that $\Phi^{*}$ is $C^{1,1}(\mathbb{R})$. In this case the following reciprocity formula, which is standard in convex analysis, holds true (see e.g. [2]):

$$
-\Phi^{*}(z)+z \Phi^{* \prime}(z)=\Phi\left(\Phi^{* \prime}(z)\right)
$$

Let us consider, for $\mu \in(0,1)$ the function $w=\mu u-v$. Then $w$ satisfies in the viscosity sense

$$
\begin{cases}w_{t}-(1-\mu) \Phi\left(\frac{1}{1-\mu} H_{0}(x, D w)\right) \leq 0 & \text { in } \mathbb{R}^{N} \times(0, T] \\ w(x, 0) \leq(1-\mu) K(1+f(d(x))) & \text { in } \mathbb{R}^{N} .\end{cases}
$$

The initial condition is trivially satisfied. As far as the equation is concerned, we take $\phi \in C^{1}\left(\mathbb{R}^{N} \times[0, T]\right)$ and $(\bar{x}, \bar{t}) \in \mathbb{R}^{N} \times(0, T]$ a strict maximum of $w-\phi$ in $\bar{B}(\bar{x}, r) \times[\bar{t}-r, \bar{t}+r]$ for some $r>0$. For all $\varepsilon>0$ we set

$$
\psi_{\varepsilon}(x, y, t)=\phi(x, t)+\frac{|x-y|^{2}}{\varepsilon^{2}}
$$


and consider

$$
M_{\varepsilon}:=\max _{x, y \in \bar{B}(\bar{x}, r), t \in[\bar{t}-r, \bar{t}+r]}\left\{\mu u(x, t)-v(y, t)-\psi_{\varepsilon}(x, y, t)\right\} .
$$

This maximum is achieved at a point $\left(x_{\varepsilon}, y_{\varepsilon}, t_{\varepsilon}\right)$ and, since $(\bar{x}, \bar{t})$ is a strict maximum of $w-\phi$, standard arguments show that as $\varepsilon \rightarrow 0$,

$$
\frac{\left|x_{\varepsilon}-y_{\varepsilon}\right|^{2}}{\varepsilon^{2}} \rightarrow 0
$$

and

$$
M_{\varepsilon}=\mu u\left(x_{\varepsilon}, t_{\varepsilon}\right)-v\left(y_{\varepsilon}, t_{\varepsilon}\right)-\psi_{\varepsilon}\left(x_{\varepsilon}, y_{\varepsilon}, t_{\varepsilon}\right) \longrightarrow \mu u(\bar{x}, \bar{t})-v(\bar{x}, \bar{t})-\phi(\bar{x}, \bar{t})=w(\bar{x}, \bar{t})-\phi(\bar{x}, \bar{t}) .
$$

Moreover there are $a_{1}, a_{2} \in \mathbb{R}$ such that if $p_{\varepsilon}=\frac{2\left(x_{\varepsilon}-y_{\varepsilon}\right)}{\varepsilon^{2}}$

$$
\begin{aligned}
& \left(a_{1}, p_{\varepsilon}+D_{x} \phi\left(x_{\varepsilon}, t_{\varepsilon}\right)\right) \in D^{+} \mu u\left(x_{\varepsilon}, t_{\varepsilon}\right) \\
& \left(a_{2}, p_{\varepsilon}\right) \in D^{-} v\left(y_{\varepsilon}, t_{\varepsilon}\right) ; \\
& a_{1}-a_{2}=\frac{\partial}{\partial t} \psi_{\varepsilon}\left(x_{\varepsilon}, y_{\varepsilon}, t_{\varepsilon}\right)=\frac{\partial}{\partial t} \phi\left(x_{\varepsilon}, t_{\varepsilon}\right) \\
& a_{1}+\mu \Phi\left(\frac{1}{\mu} H_{0}\left(x_{\varepsilon}, p_{\varepsilon}+D_{x} \phi\left(x_{\varepsilon}, t_{\varepsilon}\right)\right) \leq 0\right.
\end{aligned}
$$

and

$$
a_{2}+\Phi\left(H_{0}\left(y_{\varepsilon}, p_{\varepsilon}\right)\right) \geq 0 .
$$

Thus by subtracting (2.26) to (2.25), using the convexity of $\Phi$ and $H_{0}$, the homogeneity of $H_{0}$ and taking into account that $\Phi$ is non decreasing and that

$$
\left|H_{0}\left(x_{\varepsilon}, p_{\varepsilon}\right)-H_{0}\left(y_{\varepsilon}, p_{\varepsilon}\right)\right| \leq L_{r}\left|p_{\varepsilon}\right|\left|x_{\varepsilon}-y_{\varepsilon}\right|=o_{\varepsilon}(1)
$$

as $\varepsilon \rightarrow 0$ (by $(\mathbf{H} \mathbf{1})$ and $(2.23))$, we get

$$
\begin{aligned}
0 & \geq \phi_{t}\left(x_{\varepsilon}, t_{\varepsilon}\right)+\mu \Phi\left(\frac{1}{\mu} H_{0}\left(x_{\varepsilon}, p_{\varepsilon}+D_{x} \phi\left(x_{\varepsilon}, t_{\varepsilon}\right)\right)-\Phi\left(H_{0}\left(y_{\varepsilon}, p_{\varepsilon}\right)\right)\right. \\
& \geq \phi_{t}\left(x_{\varepsilon}, t_{\varepsilon}\right)-(1-\mu) \Phi\left(\frac{H_{0}\left(y_{\varepsilon}, p_{\varepsilon}\right)-H_{0}\left(x_{\varepsilon}, p_{\varepsilon}+D_{x} \phi\left(x_{\varepsilon}, t_{\varepsilon}\right)\right)}{1-\mu}\right) \\
& \geq \phi_{t}\left(x_{\varepsilon}, t_{\varepsilon}\right)-(1-\mu) \Phi\left(\frac{H_{0}\left(y_{\varepsilon}, p_{\varepsilon}\right)+H_{0}\left(x_{\varepsilon}, D_{x} \phi\left(x_{\varepsilon}, t_{\varepsilon}\right)\right)-H_{0}\left(x_{\varepsilon}, p_{\varepsilon}\right)}{1-\mu}\right) \\
& \geq \phi_{t}\left(x_{\varepsilon}, t_{\varepsilon}\right)-(1-\mu) \Phi\left(\frac{H_{0}\left(x_{\varepsilon}, D_{x} \phi\left(x_{\varepsilon}, t_{\varepsilon}\right)\right)+o_{\varepsilon}(1)}{1-\mu}\right) .
\end{aligned}
$$

By letting $\varepsilon \rightarrow 0$ in $(2.27)$ we get

$$
\phi_{t}(\bar{x}, \bar{t})-(1-\mu) \Phi\left(\frac{1}{1-\mu} H_{0}\left(\bar{x}, D_{x} \phi(\bar{x}, \bar{t})\right) \leq 0 .\right.
$$

Since $H_{0} \leq \mathrm{CH}_{1}$, we obtain that $w$ is a subsolution also of

$$
w_{t}-(1-\mu) \Phi\left(\frac{C}{1-\mu} H_{1}\left(x, D_{x} w\right)\right) \leq 0
$$


Now we find a viscosity supersolution $\chi_{\delta}(x, t)$ of $(2.28)$ satisfying $\chi_{\delta}(x, 0) \geq(1-\mu) K(1+f(d(x)))$. To this purpose we consider the function

$$
\chi_{\delta}(x, t)=(1-\mu)\left[\frac{K^{\prime}}{1-4 M t}+\Phi^{*}\left(\frac{(d(x)-\delta)^{+}}{C(1-4 M t)}\right)(1-4 M t)\right]
$$

where $K^{\prime}, M$ are positive constants to be chosen later and $d(x)=d(x ; 0)$ is the viscosity solution of $(0.6)$. The following estimates hold

$$
\frac{\partial}{\partial t} \chi_{\delta}=(1-\mu)\left[\frac{4 M K^{\prime}}{(1-4 M t)^{2}}-4 M \Phi^{*}\left(\frac{(d(x)-\delta)^{+}}{C(1-4 M t)}\right)+\Phi^{* \prime}\left(\frac{(d(x)-\delta)^{+}}{C(1-4 M t)}\right) \frac{4 M(d(x)-\delta)^{+}}{C(1-4 M t)}\right]
$$

and, being $H_{1}$ homogeneous of degree 1 with respect to $p$,

$$
\Phi\left(\frac{C}{1-\mu} H_{1}\left(x, D_{x}^{-} \chi_{\delta}\right)\right)=\Phi\left(\Phi^{* \prime}\left(\frac{(d(x)-\delta)^{+}}{C(1-4 M t)}\right) H_{1}\left(x, D^{-} d(x)\right)\right) .
$$

Moreover, since $d$ is a solution of the convex equation (0.6), we have by Theorem 2.1 that $H_{1}\left(x, D^{-} d(x)\right)=1$. Therefore, putting

we obtain

$$
z=\frac{(d(x)-\delta)^{+}}{C(1-4 M t)}
$$

$$
\begin{aligned}
\frac{\partial}{\partial t} \chi_{\delta}-(1-\mu) \Phi\left(\frac{C}{1-\mu} H_{1}\left(x, D_{x}^{-} \chi_{\delta}\right)\right)= & \frac{(1-\mu) 4 M K^{\prime}}{(1-4 M t)^{2}}+4 M(1-\mu)\left(z \Phi^{* \prime}(z)-\Phi^{*}(z)\right) \\
& -(1-\mu) \Phi\left(\Phi^{* \prime}(z)\right) \\
= & (1-\mu)\left[\frac{4 M K^{\prime}}{(1-4 M t)^{2}}+(4 M-1) \Phi\left(\Phi^{* \prime}(z)\right)\right],
\end{aligned}
$$

where we use in the last equality the formula (2.21). Thus if $M>\frac{1}{4}$ and $t<\frac{1}{4 M}$ we get

$$
\frac{\partial}{\partial t} \chi_{\delta}-(1-\mu) \Phi\left(\frac{C}{1-\mu} H_{1}\left(x, D_{x}^{-} \chi_{\delta}\right)\right)>0
$$

and we prove the claim.

We have

$$
\left.\chi_{\delta}(x, 0)\right)=(1-\mu)\left[K^{\prime}+\Phi^{*}\left(\frac{(d(x)-\delta)^{+}}{C}\right)\right] .
$$

Since $\Phi^{*}$ is nondecreasing, $\frac{\Phi^{*}(d(x))}{f(d(x))} \rightarrow+\infty$ and $f\left(\frac{1-\delta}{C} d(x)\right) \sim L f(d(x))$, as $d(x) \rightarrow \infty$, we can find $K^{\prime}$ large enough so that

$$
\chi_{\delta}(x, 0) \geq(1-\mu) K(1+f(d(x))) .
$$

Next for all $\varepsilon>0$, we introduce the auxiliary function

$$
\Phi_{\varepsilon, \eta}(x, y, t)=w(x, t)-\chi_{\delta}(y, t)-\eta t-\frac{|x-y|^{\gamma}}{\gamma \varepsilon},
$$

$\gamma$ being the constant appearing in (0.13). By arguing as in the proof of Theorem 2.2, one can show that $\max _{\mathbb{R}^{N} \times \mathbb{R}^{N} \times[0, \tau]} \Phi_{\varepsilon, \eta}(x, y, t)$ is achieved at a point $\left(x_{\varepsilon}, y_{\varepsilon}, t_{\varepsilon}\right)$, lying in a compact set independent of $\varepsilon$ such that 
(up to subsequences) as $\varepsilon \rightarrow 0$

$$
\begin{aligned}
& \frac{\left|x_{\varepsilon}-y_{\varepsilon}\right|^{\gamma}}{\gamma \varepsilon} \rightarrow 0, \quad t_{\varepsilon} \rightarrow \bar{t}>0 \\
& \Phi_{\varepsilon}\left(x_{\varepsilon}, y_{\varepsilon}, t_{\varepsilon}\right) \rightarrow M_{\eta} .
\end{aligned}
$$

Thus there are $a_{1}, a_{2} \in \mathbb{R}$ such that such that if $p_{\varepsilon}=\left|x_{\varepsilon}-y_{\varepsilon}\right|^{\gamma-2} \frac{\left(x_{\varepsilon}-y_{\varepsilon}\right)}{\varepsilon}$

$$
\begin{aligned}
&\left(a_{1}, p_{\varepsilon}\right) \in D^{+} w\left(x_{\varepsilon}, t_{\varepsilon}\right) ; \\
&\left(a_{2}, p_{\varepsilon}\right) \in D^{-} \chi_{\delta}\left(y_{\varepsilon}, t_{\varepsilon}\right) ; \\
& a_{1}-a_{2}=\eta ; \\
& a_{1}-(1-\mu) \Phi\left(\frac{C}{1-\mu} H_{1}\left(x_{\varepsilon}, p_{\varepsilon}\right)\right) \leq 0
\end{aligned}
$$

and

$$
a_{2}-(1-\mu) \Phi\left(\frac{C}{1-\mu} H_{1}\left(y_{\varepsilon}, p_{\varepsilon}\right)\right) \geq 0 .
$$

We first observe that $\left|x_{\varepsilon}-y_{\varepsilon}\right|\left|p_{\varepsilon}\right| \rightarrow 0$ as $\varepsilon \rightarrow 0$. Moreover, since $p_{\varepsilon} \in D^{-} \chi_{\delta}\left(y_{\varepsilon}, t_{\varepsilon}\right)$, by Theorem 2.1, for some $q_{\varepsilon} \in D^{-} d\left(y_{\varepsilon}\right)$ and for some $C^{\prime}>0$ we have

$$
H_{1}\left(y_{\varepsilon}, p_{\varepsilon}\right)=\frac{(1-\mu)}{C} \Phi^{* \prime}\left(\frac{\left(d\left(y_{\varepsilon}\right)-\delta\right)^{+}}{C\left(1-4 M t_{\varepsilon}\right)}\right) H_{1}\left(y_{\varepsilon}, q_{\varepsilon}\right) \leq C^{\prime} .
$$

Hence, from (0.3) satisfied by $H_{1}$, we have that $\left|H_{1}\left(x_{\varepsilon}, p_{\varepsilon}\right)\right|$ is bounded as well and moreover,

$$
\left|H_{1}\left(x_{\varepsilon}, p_{\varepsilon}\right)-H_{1}\left(y_{\varepsilon}, p_{\varepsilon}\right)\right|=o_{\varepsilon}(1) \text { as } \varepsilon \rightarrow 0 .
$$

Thus, in view of the continuity of $\Phi$ we get

$$
\left|\Phi\left(\frac{C}{1-\mu} H_{1}\left(x_{\varepsilon}, p_{\varepsilon}\right)\right)-\Phi\left(\frac{C}{1-\mu} H_{1}\left(y_{\varepsilon}, p_{\varepsilon}\right)\right)\right|=o_{\varepsilon}(1),
$$

as $\varepsilon \rightarrow 0$. Thus by subtracting (2.31) to (2.32) we get

$$
0<\eta \leq(1-\mu) \Phi\left(\frac{C}{1-\mu} H_{1}\left(x_{\varepsilon}, p_{\varepsilon}\right)\right)-(1-\mu) \Phi\left(\frac{C}{1-\mu} H_{1}\left(y_{\varepsilon}, p_{\varepsilon}\right)\right)=o_{\varepsilon}(1) .
$$

By letting $\varepsilon \rightarrow 0$ we get a contradiction and we conclude.

Step 2. In the general case we introduce for all $\lambda>0$ the function

$$
\Phi^{\lambda}(s)=\Phi(s)+\frac{s^{2}}{2 \lambda}
$$

We observe that

$$
\Phi_{\lambda}(s)=\frac{|s|^{2}}{2 \lambda}-\left(\Phi(s)+\frac{s^{2}}{2 \lambda}\right)^{*}
$$

where $\Phi_{\lambda}(s)$ denotes the inf-convolution of $\Phi$. It is well-known that $\Phi_{\lambda}(s)$ is in $C^{1,1}(\mathbb{R})$, being $\Phi$ a convex function (see e.g. Attouch [2]). Thus $\Phi^{\lambda}(s)$ is in $C^{1,1}(\mathbb{R})$ as well and moreover $\left(\Phi^{\lambda}\right)^{*}$ is strictly increasing, see $[16]$. 
Now it is sufficient to notice that the function $w=\mu u-v$ satisfies in the viscosity sense also the equation

$$
w_{t}-(1-\mu) \Phi^{\lambda}\left(\frac{1}{1-\mu} H_{0}(x, D w)\right) \leq 0
$$

The conclusion follows by applying Step 1.

We remark that since $(0.4)$ implies $H(x,-p)=H(x, p)$, then from Theorem 2.3 it follows directly the following comparison principle for concave Hamiltonians.

Theorem 2.4. Assume (H1), (H2) and that $h \in C\left(\mathbb{R}^{N} ; \mathbb{R}\right)$ satisfies $h \leq K(1+f(d(x)))$ for some $K>0$, where $d(x)=d(x ; 0)$ is defined in $(0.6)$. Let $u \in U S C\left(\mathbb{R}^{N} \times[0, T)\right)$ and $v \in L S C\left(\mathbb{R}^{N} \times[0, T)\right)$ be respectively a viscosity sub- and super-solution of the problem

$$
\begin{cases}u_{t}(x, t)-H(x, D u)=0 & \text { in } \mathbb{R}^{N} \times(0, T) \\ u(x, 0)=h(x) & \text { in } \mathbb{R}^{N} .\end{cases}
$$

Assume that $u(x, t) \leq K(1+f(d(x)))$. Then $u(x, t) \leq v(x, t)$ in $\mathbb{R}^{N} \times[0, T]$.

Remark 2.1. In the case where $\Phi(t)=t^{\beta}$, for some $\beta>1$, then we can choose $f(t)=t^{\alpha}$ with $0<\alpha<\frac{\beta}{\beta-1}$ provided that the solution $d$ of $(0.6)$ satisfies $d(x ; y)=o\left(|x-y|^{\frac{\gamma}{\alpha}}\right)$ for $|x-y| \rightarrow+\infty$, for some $\gamma>1$. This holds true in particular for the distances associated to Suplaplacians, which behaves at infinity as $|x-y|$.

It will be the aim of future investigation, the limit case $f(d(x ; y))=O\left(\Phi^{*}(d(x ; y))\right)$. At this purpose, let us observe that if $\Phi(t)=t \log (1+t)$ and $d(x ; y) \sim|x-y|$ at infinity, then $\Phi^{*}(t) \geq t^{\frac{\alpha}{\alpha-1}}$ for all $\alpha>1$, so that we cannot find functions $f$ satisfying both the conditions: $f(|x-y|)-|x-y|^{\gamma} \rightarrow-\infty$ as $|x-y| \rightarrow+\infty$, for some $\gamma>1$ and $f(d)=O\left(\Phi^{*}(d)\right)$. Indeed, choosing $\alpha<\frac{\gamma}{\gamma-1}$, we get

$$
\frac{f(d)}{\Phi^{*}(d)}=\frac{f(d)}{|x-y|^{\gamma}} \frac{|x-y|^{\gamma}}{\Phi^{*}(d)} \leq \frac{|x-y|^{\gamma}}{\Phi^{*}(d)} \leq \frac{|x-y|^{\gamma}}{|x-y|^{\alpha-1}} \rightarrow 0 .
$$

\section{EXISTENCE RESUlts}

In the framework of viscosity solutions, the existence is usually obtained as a consequence of the comparison principle by means of Perron's method of Ishii [22] as soon as we can build a sub- and a supersolution to the problem (0.1) which are bounded from below by a function growing at most as $f(d(x)), f(t)$ being the function appearing in (0.13).

As mentioned in the introduction, if $H_{0}$ satisfies (0.6) (so that we can choose $H_{1}=H_{0}$ ) the existence of a solution of (0.1) has been proved by Capuzzo Dolcetta and Ishii in [16], (see also [14,15]). Moreover, they provide the following Hopf-type representation formula for the solution of $(0.1)$ with $t \in[0,+\infty)$ :

$$
u(x, t)=\inf _{y \in \mathbb{R}^{N}}\left[h(y)+t \Phi^{*}\left(\frac{d(x ; y)}{t}\right)\right],
$$

where $d(x ; y)$ is the distance associated to $H_{0}$. The following Theorem provides an existence result in the case where $H_{0}$ does not satisfies (0.6) and therefore the result of [16] does not apply.

Theorem 3.1 (existence convex case). Assume (H1), (H2) and suppose that $h(x) \geq-K(1+f(d(x)))$ for some $K>0$, where $d(x)=d(x ; 0)$ is defined in $(0.6)$. Then there is a unique viscosity continuous solution $u(x, t)$ of $(0.1)$ such that $u(x, t) \geq-K(1+f(d(x)))$ for all $(x, t) \in \mathbb{R}^{N} \times[0, T]$.

Proof. The function

$$
\underline{u}(x, t)=\inf _{y \in \mathbb{R}^{N}}\left[h(y)+t \Phi^{*}\left(\frac{d(x ; y)}{C t}\right)\right]
$$


is a viscosity subsolution of $(0.1)$ since $\Phi\left(H_{0}(x, p)\right) \leq \Phi\left(C H_{1}(x, p)\right)$ and $u$ is a viscosity solution of $(0.1)$ with $H(x, p)=\Phi\left(C H_{1}(x, p)\right)$ as it is proved in [16]. Moreover, for all $M>0$, the function $\bar{u}(x, t)=M t+h(x)$ is a viscosity supersolution as it is immediate to check simply by observing that $H(x, p) \geq 0$ for all $x, p$. Since $\underline{u}(x, t) \leq \bar{u}(x, t)$, by the Perron method (see [22]), there exists a continuous solution of $(0.1)$ in between $\underline{u}$ and $\bar{u}$ and we conclude.

Since $H(x,-p)=H(x, p)$, from Theorem 3.1 it follows directly an existence result for the concave problem (2.34). More precisely,

Theorem 3.2 (existence concave case). Assume (H1), (H2) and suppose that $h(x) \leq K(1+f(d(x)))$ for some $K>0$, where $d(x)=d(x ; 0)$ is defined in $(0.6)$. Then there is a unique continuous viscosity solution $u(x, t)$ of (2.34) such that $u(x, t) \leq K(1+f(d(x)))$ for all $(x, t) \in \mathbb{R}^{N} \times[0, T]$.

We observe that Theorem 3.2 can be proved independently from Theorem 3.1 by using as barriers the functions $\underline{u}(x, t)=-M t+h(x)$ which is a subsolution of (2.34) for $M>0$ large enough and

$$
\bar{u}(x, t)=K+3 T\left(1-\frac{t}{2 T}\right) \Phi^{*}\left(\frac{(d(x)-\delta)^{+}}{3 C T\left(1-\frac{t}{2 T}\right)}\right)
$$

which is supersolutions of (2.34) if $\delta \in(0,1), K>0$ is large enough, and $C>0$ is the constant defined in (H2).

Acknowledgements. The authors would like to thank Prof. Guy Barles and Prof. Italo Capuzzo Dolcetta for useful discussions and comments and Prof. Franco Rampazzo for suggesting the example 0.1.

This work was partially supported by M.I.U.R., project "Viscosity, metric, and control theoretic methods for nonlinear partial differential equations", by I.N.D.A.M. intergroup project "Controllo e numerica" and by G.N.A.M.P.A, project "Equazioni alle derivate parziali e teoria del controllo".

\section{REFERENCES}

[1] O. Alvarez, Bounded-from-below viscosity solutions of Hamilton-Jacobi equations. Differential Integral Equations 10 (1997) 419-436.

[2] H. Attouch, Variational convergence for functions and operators. Applicable Mathematics Series, Pitman (Advanced Publishing Program), Boston, MA (1984).

[3] M. Bardi and I. Capuzzo Dolcetta, Optimal control and viscosity solutions of Hamilton-Jacobi-Bellman equations. Birkhäuser, Boston (1997)

[4] M. Bardi and F. Da Lio, On the Bellman equation for some unbounded control problems. NoDEA 4 (1997) 491-510.

[5] G. Barles, Solutions de viscosité des équations de Hamilton-Jacobi. Springer-Verlag, Paris (1994).

[6] E.N. Barron and R. Jensen, Generalized viscosity solutions for Hamilton-Jacobi equations with time-measurable Hamiltonians. J. Differential Equations 68 (1987) 10-21.

[7] E.N. Barron and R. Jensen, Semicontinuous viscosity solutions for Hamilton-Jacobi equations with convex hamiltonians. Commun. Partial Differ. Equ. 15 (1990) 1713-1742.

[8] A. Bellaiche and J.-J. Risler, Sub-Riemannian geometry, Progress in Mathematics 144, Birkhäuser Verlag, Basel (1996).

[9] A. Bensoussan, Stochastic control by functional analysis methods, Studies in Mathematics and its Applications 11, NorthHolland Publishing Co., Amsterdam (1982)

[10] I. Birindelli and J. Wigniolle, Homogenization of Hamilton-Jacobi equations in the Heisenberg group. Commun. Pure Appl. Anal. 2 (2003) 461-479.

[11] R.W. Brockett, Control theory and singular Riemannian geometry, in: New Directions in Applied Mathematics (Cleveland, Ohio, 1980) Springer, New York-Berlin (1982) 11-27.

[12] R.W. Brockett, Pattern generation and the control of nonlinear systems. IEEE Trans. Automatic Control 48 (2003) 1699-1711.

[13] P. Cannarsa and G. Da Prato, Nonlinear optimal control with infinite horizon for distributed parameter systems and stationary Hamilton-Jacobi equations. SIAM J. Control Optim. 27 (1989) 861-875.

[14] I. Capuzzo Dolcetta, The Hopf solution of Hamilton-Jacobi equations. Elliptic and parabolic problems (Rolduc/Gaeta) (2001) 343-351.

[15] I. Capuzzo Dolcetta, Representations of solutions of Hamilton-Jacobi equations. Progr. Nonlinear Differential Equations Appl. $\mathbf{5 4}(2003)$ 79-90.

[16] I. Capuzzo Dolcetta and H. Ishii, Hopf formulas for state-dependent Hamilton-Jacobi equations. Preprint. 
[17] A. Cutrì, Problemi semilineari ed integro-differenziali per sublaplaciani. Ph.D. Thesis, Universitá di Roma Tor Vergata (1997).

[18] F. Da Lio and O. Ley, Uniqueness Results for Second Order Bellman-Isaacs Equations under Quadratic Growth Assumptions and Applications, Quaderno 8, Dipartimento di Matematica, Università di Torino (2004).

[19] F. Da Lio and W.M. McEneaney, Finite time-horizon risk-sensitive control and the robust limit under a quadratic growth assumption. SIAM J. Control Optim 40 (2002) 1628-1661 (electronic).

[20] C.L. Fefferman and D.H. Phong, Subelliptic eigenvalue problems, in Conference on Harmonic Analysis in Honor of A. Zygmund, Wadsworth Math. Series 2 (1983) 590-606 .

[21] L. Hörmander, Hypoelliptic second order differential equations. Acta Math. 119 (1967) 147-171.

[22] H. Ishii, Perron's method for Hamilton-Jacobi equations. Duke Math. J. 55 (1987) 369-384.

[23] H. Ishii, Comparison results for Hamilton-Jacobi equations without growth condition on solutions from above. Appl. Anal. 67 (1997) 357-372.

[24] D. Jerison and A. Sànchez-Calle, Subelliptic second order differential operator. Lect. Notes Math. Berlin-Heidelberg-New York 1277 (1987) 46-77.

[25] J.J. Manfredi and B. Stroffolini, A version of the Hopf-Lax formula in the Heisenberg group. Comm. Partial Differ. Equ. 27 (2002) 1139-1159.

[26] R. Monti and F. Serra Cassano, Surface measures in Carnot Caratheodory spaces. Calc. Var. Partial Differ. Equ. 13 (2001) $339-376$.

[27] A. Nagel, E.M. Stein and S. Wainger, Balls and metrics defined by vector fields. I: Basic properties. Acta Math. 155 (1985) $103-147$.

[28] F. Rampazzo and C. Sartori, Hamilton-Jacobi-Bellman equations with fast gradient-dependence. Indiana Univ. Math. J. 49 (2000) 1043-1077.

[29] F. Rampazzo and H. Sussmann, Set-valued differentials and a nonsmooth version of Chow's theorem, in Proceedings of the 40th IEEE Conference on Decision and Control, Orlando, Florida (IEEE Publications, New York, 2001) 3 (2001) $2613-2618$.

[30] B. Stroffolini, Homogenization of Hamilton-Jacobi Equations in Carnot Groups. ESAIM: COCV 13 (2007) $107-119$.

[31] H.J. Sussmann, A general theorem on local controllability. SIAM J. Control. Optim. 25 (1987) 158-194. 